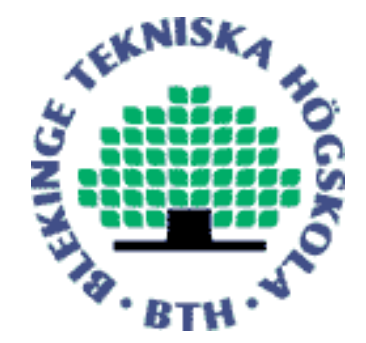

Copyright (C) 2012 IEEE.

Citation for the published paper:

MRT/MRC for Cognitive AF Relay Networks under Feedback Delay and Channel Estimation Error

Thi My Chinh Chu, Quang Trung Duong, Hans-Jürgen Zepernick

IEEE International Symposium on Personal, Indoor and Mobile Radio Communications (PIMRC)

2012 Sydney, Australia

This material is posted here with permission of the IEEE. Such permission of the IEEE does not in any way imply IEEE endorsement of any of BTH's products or services Internal or personal use of this material is permitted. However, permission to reprint/republish this material for advertising or promotional purposes or for creating new collective works for resale or redistribution must be obtained from the IEEE by sending a blank email message to pubs-permissions@iee.org.

By choosing to view this document, you agree to all provisions of the copyright laws protecting it. 


\title{
MRT/MRC for Cognitive AF Relay Networks under Feedback Delay and Channel Estimation Error
}

\author{
Thi My Chinh Chu, Trung Q. Duong, and Hans-Jürgen Zepernick \\ Blekinge Institute of Technology, SE-371 79 Karlskrona, Sweden \\ E-mail: $\{$ cch, dqt, hjz $\} @$ bth.se
}

\begin{abstract}
In this paper, we examine the performance of multiple-input multiple-output (MIMO) cognitive amplify-andforward (AF) relay systems with maximum ratio transmission (MRT). In particular, closed-form expressions in terms of a tight upper bound for outage probability (OP) and symbol error rate (SER) of the system are derived when considering channel estimation error (CEE) and feedback delay (FD) in our analysis. Through our works, one can see the impact of FD and CEE on the system as well as the benefits of deploying multiple antennas at the transceivers utilizing the spatial diversity of an MRT system. Finally, we also provide a comparison between analytical results and Monte Carlo simulations for some examples to verify our work.
\end{abstract}

\section{INTRODUCTION}

Two major challenges for a communication system design are how to use frequency resources efficiently and how to overcome multipath fading to guarantee transmission reliability. Recently, studies on cooperative diversity techniques and cognitive radio networks (CRNs) have revealed promising solutions to combat these difficulties. Deploying cooperative relaying has been shown to offer many benefits such as extending coverage, improving throughput and enhancing transmission reliability [1], [2]. Moreover, CRNs permit unlicensed users, also called secondary users, to access the licensed spectrum opportunistically or concurrently as reported in [3]. Therefore, combining cooperative diversity with CRNs not only improves the efficiency of spectrum usage but also provides higher transmission reliability, e.g. [1]-[4]. Specifically, the works of [3] presented a brief overview about the cognitive cooperative techniques. Further, [1], [2], [4] obtained improved throughput of secondary nodes by increasing spatial diversity through a cooperative cognitive approach.

When considering techniques which enable a secondary user to access licensed spectrum, there are two kinds of approaches, spectrum underlay and spectrum overlay. In spectrum overlay, a secondary user is only allowed to use licensed spectrum when the primary user is idle. Whenever the primary user becomes active, the overlay secondary user must switch off its transmission and search for another spectrum hole. Hence, there is no constraint on transmit power at the secondary transmitter in the overlay approach. Instead, spectrum sensing and detecting a spectrum white space are required at the secondary user. Specifically, [5], [6] proposed spectrum sharing schemes in decode-and-forward (DF) relay overlay cognitive networks for single relay and multiple relays, respectively. In the underlay approach, both secondary users and primary users can use the same spectrum simultaneously. As such, underlay secondary transmitters must constrain their transmit power to guarantee that the interference at the primary user is kept below a given threshold. For this reason, their coverage is often not large. If a secondary user wants to extend its coverage, it normally cooperates with other relays. In particular, [7] analyzed outage probability (OP) for a DF relay cognitive network while [8] investigated OP for an amplify-and-forward (AF) relay cognitive network. Moreover, [9] proposed a power allocation to enhance the spectral efficiency and increase the bit rate for a network coded cognitive cooperative network (NCCCN) under peak interference constraints. Additionally, [10], [11] proposed algorithms to distribute transmit power for beamforming transmission via a multi-relay underlay cognitive radio architecture. Besides beamforming transmission, maximum ratio transmission (MRT) is shown in [12] as a powerful diversity technique. Deploying antenna arrays in maximum ratio transmission has been shown to offer many benefits such as combating the adverse effect of fading, increasing capacity and extending coverage [12]. Thus, MRT seems to be suitable for underlay cognitive transmission which suffers from a very strict constraint on their transmit power. To the best of our knowledge, there is no work focusing on MRT for an underlay cognitive AF relay network.

In this paper, we deploy MRT with two hop AF relaying in an underlay cognitive network. Specifically, we derive a closed-form expression for the cumulative distribution function (CDF) of the instantaneous signal-noise-ratio (SNR). This outcome enables us to obtain a tight upper bound for the outage probability and symbol error rate (SER) of the considered system.

The paper is organized as follow: Section II describes the considered system, related concepts and definitions. The system performance in terms of outage probability and symbol error rate are analyzed in Section III. Section IV presents numerical results and discussions of the achieved results. Finally, conclusions are given in Section V.

Notation: In this paper, matrices and vectors are denoted by bold upper and lower case letters, respectively. Next, $\|.\|_{F}$ indicates the Frobenius norm and $\dagger$ stands for transpose conjugate of a vector or matrix. Then, the probability density function (PDF) and the cumulative distribution function (CDF) of a random variable $X$ are written as $f_{X}($.$) and F_{X}($.$) , respec-$ 
tively. Furthermore, the gamma function in [13, eq. (8.310.1)] and the incomplete gamma function in [13, eq. (8.350.2)] are denoted as $\Gamma(n, x)$ and $\Gamma(n)$, respectively. Finally, the confluent hypergeometric function [13, eq. (9.211.4)] is expressed by $U(a, b ; x)$.

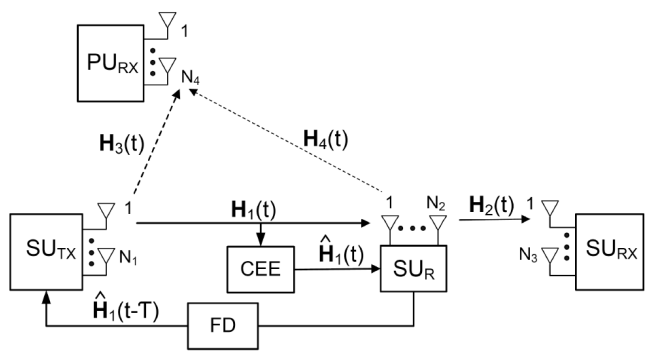

Fig. 1. System model for a cognitive MRT AF relay network under channel estimation error and feedback delay.

\section{System AND Channel Model}

We consider an underlay cognitive AF relay system including $N_{1}$ antennas at the secondary transmitter $\mathrm{SU}_{\mathrm{TX}}, N_{2}$ antennas at the secondary relay $\mathrm{SU}_{\mathrm{R}}, N_{3}$ antennas at the secondary receiver $\mathrm{SU}_{\mathrm{RX}}$ and $N_{4}$ antennas at the primary receiver $P_{\mathrm{RX}}$ as shown in Fig. 1. The primary and the secondary users (including $\mathrm{SU}_{\mathrm{TX}}$ and $\mathrm{SU}_{\mathrm{R}}$ ) can simultaneously access the same spectrum as long as the secondary users guarantee that their interference to the primary user is kept below a predefined threshold, $Q$. In the first hop from $\mathrm{SU}_{\mathrm{TX}}$ to $\mathrm{SU}_{\mathrm{R}}$, we implement MRT at the $\mathrm{SU}_{\mathrm{TX}}$ by multiplying the transmit signal, $s(t)$ with an $N_{1} \times 1$ transmit weighting vector $\mathbf{v}_{1}(t)$ and employ maximum ratio combining (MRC) at the $\mathrm{SU}_{\mathrm{R}}$ by multiplying the received signal with an $1 \times N_{2}$ receive weighting vector $\mathbf{w}_{1}(t)$. As a result, the received signal $s_{r}(t)$ at the $\mathrm{SU}_{\mathrm{R}}$ is given by

$$
s_{r}(t)=\mathbf{w}_{1}(t)\left[\mathbf{H}_{1}(t) \mathbf{v}_{1}(t) s(t)+\mathbf{n}_{1}(t)\right]
$$

where $\mathbf{H}_{1}(t)$ is an $N_{2} \times N_{1}$ channel coefficient matrix from $\mathrm{SU}_{\mathrm{TX}}$ to $\mathrm{SU}_{\mathrm{R}}$ whose elements are independent and identical distributed (i.i.d.) complex Gaussian random variables (RV) with zero mean and variance $\Omega_{1}$, denoted as $\mathcal{C N}\left(0, \Omega_{1}\right)$. Further, $s(t)$ is the transmit signal at the $\mathrm{SU}_{\mathrm{TX}}$ with average power $E\left\{|s(t)|^{2}\right\}=P_{1}$ where $E\{\cdot\}$ stands for an expectation operator. Finally, $\mathbf{n}_{1}(t)$ is an $N_{2} \times 1$ additive white Gaussian noise (AWGN) vector at the $\mathrm{SU}_{\mathrm{R}}$. It is assumed that all elements of $\mathbf{n}_{1}(t)$ are i.i.d. complex Gaussian RVs with zero mean and variance $N_{0}$, denoted as $\mathcal{C N}\left(0, N_{0}\right)$. To get maximum signal-to-noise ratio $(\mathrm{SNR})$ at the $\mathrm{SU}_{\mathrm{R}}$, the transmit weighting vector $\mathbf{v}_{1}(t)$ is chosen to be the eigenvector $\mathbf{u}_{1}(t)$ corresponding to the largest eigenvalue of the Wishart matrix $\mathbf{H}_{1}^{\dagger}(t) \mathbf{H}_{1}(t)$ and the receive weighting vector $\mathbf{w}_{1}(t)$ is selected as $\mathbf{w}_{1}(t)=\mathbf{u}_{1}^{\dagger}(t) \mathbf{H}_{1}^{\dagger}(t)$.

In order to deploy MRT/MRC, $\mathrm{SU}_{\mathrm{TX}}$ and $\mathrm{SU}_{\mathrm{R}}$ need the channel state information (CSI) to adjust the weighting vectors. However, the $\mathrm{SU}_{\mathrm{RX}}$ can usually not perfectly estimate
$\mathbf{H}_{1}(t)$ and there always exits feedback delay (FD) from $\mathrm{SU}_{\mathrm{R}}$ to $\mathrm{SU}_{\mathrm{TX}}$ in practice. When taking into account the effect of channel estimation error (CEE) and FD, $\tau$, the channel coefficient matrix at the $\mathrm{SU}_{\mathrm{TX}}$ is $\hat{\mathbf{H}}_{1}(t-\tau)$. As a consequence, $\mathbf{v}_{1}(t)$ is selected as the eigenvector $\hat{\mathbf{u}}_{1}(t)$ corresponding to the largest eigenvalue $\lambda_{\text {max }_{1}}$ of the Wishart matrix $\hat{\mathbf{H}}_{1}^{\dagger}(t-\tau) \hat{\mathbf{H}}_{1}(t-\tau)$ and $\mathbf{w}_{1}(t)$ is chosen to be $\mathbf{w}_{1}(t)=\hat{\mathbf{u}}_{1}^{\dagger}(t) \hat{\mathbf{H}}_{1}^{\dagger}(t-\tau)$. As mentioned in [14], the relationship between $\mathbf{H}_{1}(t)$ and $\hat{\mathbf{H}}_{1}(t-\tau)$ is given by

$$
\mathbf{H}_{1}(t)=\rho \hat{\mathbf{H}}_{1}(t-\tau)+\mathbf{E}(t)+\mathbf{D}(t)
$$

where $\rho$ denotes the channel correlation coefficient. As in [14], for the Clarkes fading spectrum, $\rho$ is expressed in terms of FD $\tau$ and the Doppler frequency $f_{d}$ as $\rho=J_{0}\left(2 \pi f_{d} \tau\right)$ where $J_{0}(\cdot)$ is the zero-th order Bessel function of the first kind [13, eq.(8.441.1)]. Further, $\mathbf{E}(t)$ is an $N_{2} \times N_{1}$ CEE matrix whose elements are i.i.d. complex Gaussian RVs, $\mathcal{C N}\left(0, \sigma^{2} \Omega_{1}\right) ; \sigma^{2}$ is the variance of CEE. Finally, $\mathbf{D}(t)$ stands for an $N_{2} \times N_{1}$ error matrix induced by FD whose elements are i.i.d. complex Gaussian RVs, $\mathcal{C N}\left(0,\left(1-\sigma^{2}\right)\left(1-\rho^{2}\right) \Omega_{1}\right)$. For this more practical scenario, the received signal at the $S_{R}$ is given by

$$
\begin{aligned}
\hat{s}_{r}(t) & =\rho \hat{\mathbf{u}}_{1}^{\dagger}(t) \hat{\mathbf{H}}_{1}^{\dagger}(t-\tau) \hat{\mathbf{H}}_{1}(t-\tau) \hat{\mathbf{u}}_{1}(t) s(t)+\hat{\mathbf{u}}_{1}^{\dagger}(t) \hat{\mathbf{H}}_{1}^{\dagger}(t-\tau) \\
& \times \mathbf{E}(t) \hat{\mathbf{u}}_{1}(t) s(t)+\hat{\mathbf{u}}_{1}^{\dagger}(t) \hat{\mathbf{H}}_{1}^{\dagger}(t-\tau) \mathbf{D}(t) \hat{\mathbf{u}}_{1}(t) s(t) \\
& +\hat{\mathbf{u}}_{1}^{\dagger}(t) \hat{\mathbf{H}}_{1}^{\dagger}(t-\tau) \mathbf{n}_{1}(t)
\end{aligned}
$$

At the $\mathrm{SU}_{\mathrm{R}}$, the received signal is amplified with a factor $G$ and is then forwarded to the $\mathrm{SU}_{\mathrm{RX}}$. Let $P_{2}$ be the average transmit signal at $\mathrm{SU}_{\mathrm{R}}$, the gain factor $G$ must satisfy $E\left\{\left|G \hat{s}_{r}(t)\right|^{2}\right\}=P_{2}$ or

$$
G^{2} \approx \frac{P_{2}}{\rho^{2} \lambda_{\text {max }_{1}}^{2} P_{1}}
$$

Due to the interference constraint $Q$ at the $\mathrm{PU}_{\mathrm{RX}}$, both $\mathrm{SU}_{\mathrm{TX}}$ and $\mathrm{SU}_{\mathrm{R}}$ must control their transmit power $P_{1}$, and $P_{2}$, respectively, to meet the power interference constraint at $\mathrm{PU}_{\mathrm{RX}}$, i.e.,

$$
\begin{aligned}
P_{1} & =\frac{Q}{\left\|\mathbf{H}_{3}(t)\right\|_{F}^{2}} \\
P_{2} & =\frac{Q}{\left\|\mathbf{H}_{4}(t)\right\|_{F}^{2}}
\end{aligned}
$$

where $\mathbf{H}_{3}(t)$ stands for an $N_{4} \times N_{1}$ fading channel matrix from $\mathrm{SU}_{\mathrm{TX}}$ to $\mathrm{PU}_{\mathrm{RX}}$, and $\mathbf{H}_{4}(t)$ denotes an $N_{4} \times N_{2}$ fading channel matrix from $\mathrm{SU}_{\mathrm{R}}$ to $\mathrm{PU}_{\mathrm{RX}}$. It is assumed that all elements of $\mathbf{H}_{3}(t)$ are i.i.d. complex Gaussian RVs with zero mean and variance $\Omega_{3}, \mathcal{C N}\left(0, \Omega_{3}\right)$; and all elements of $\mathbf{H}_{4}(t)$ are i.i.d. complex Gaussian RVs with zero mean and variance $\Omega_{4}$, $\mathcal{C N}\left(0, \Omega_{4}\right)$.

In the second hop, we also deploy MRT at the $\mathrm{SU}_{\mathrm{R}}$ with an $N_{2} \times 1$ transmit weighting vector $\mathbf{v}_{2}(t)$ and $\mathrm{MRC}$ at the $\mathrm{SU}_{\mathrm{RX}}$ with an $1 \times N_{3}$ receive weighting vector $\mathbf{w}_{2}(t)$. For this hop, $\mathbf{v}_{2}(t)$ is selected to be the eigenvector $\mathbf{u}_{2}(t)$ corresponding to the largest eigenvalue $\lambda_{\max _{2}}$ of the Wishart matrix 
$\mathbf{H}_{2}^{\dagger}(t) \mathbf{H}_{2}(t)$, and $\mathbf{w}_{2}(t)$ is chosen as $\mathbf{w}_{2}(t)=\mathbf{u}_{2}^{\dagger}(t) \mathbf{H}_{2}^{\dagger}(t)$. Here, $\mathbf{H}_{2}(t)$ is an $N_{3} \times N_{2}$ fading channel matrix from $\mathrm{SU}_{\mathrm{R}}$ to $\mathrm{SU}_{\mathrm{RX}}$. Consequently, the received signal at $\mathrm{SU}_{\mathrm{RX}}$ is obtained as

$$
\begin{aligned}
& s_{D}(t)= \\
& \underbrace{G \rho \mathbf{u}_{2}^{\dagger}(t) \mathbf{H}_{2}^{\dagger}(t) \mathbf{H}_{2}(t) \mathbf{u}_{2}(t) \hat{\mathbf{u}}_{1}^{\dagger}(t) \hat{\mathbf{H}}_{1}^{\dagger}(t-\tau) \hat{\mathbf{H}}_{1}(t-\tau) \hat{\mathbf{u}}_{1}(t) s(t)}_{\text {desired signal }} \\
& +\underbrace{G \mathbf{u}_{2}^{\dagger}(t) \mathbf{H}_{2}^{\dagger}(t) \mathbf{H}_{2}(t) \mathbf{u}_{2}(t) \hat{\mathbf{u}}_{1}^{\dagger}(t) \hat{\mathbf{H}}_{1}^{\dagger}(t-\tau) \mathbf{E}(t) \hat{\mathbf{u}}_{1}(t) s(t)}_{\text {self } \text { interference }} \\
& +\underbrace{G \mathbf{u}_{2}^{\dagger}(t) \mathbf{H}_{2}^{\dagger}(t) \mathbf{H}_{2}(t) \mathbf{u}_{2}(t) \hat{\mathbf{u}}_{1}^{\dagger}(t) \hat{\mathbf{H}}_{1}^{\dagger}(t-\tau) \mathbf{D}(t) \hat{\mathbf{u}}_{1}(t) s(t)}_{\text {self interference }} \\
& +\underbrace{G \mathbf{u}_{2}^{\dagger}(t) \mathbf{H}_{2}^{\dagger}(t) \mathbf{H}_{2}(t) \mathbf{u}_{2}(t) \hat{\mathbf{u}}_{1}^{\dagger}(t) \hat{\mathbf{H}}_{1}^{\dagger}(t-\tau) \mathbf{n}_{1}(t)+\mathbf{u}_{2}^{\dagger}(t)}_{\text {noise }} \\
& \times \underbrace{\mathbf{H}_{2}^{\dagger}(t) \mathbf{n}_{2}(t)}_{\text {noise }}
\end{aligned}
$$

Here, $\mathbf{n}_{2}(t)$ is an $N_{3} \times 1$ AWGN vector at the $\mathrm{SU}_{\mathrm{R}}$ whose elements are i.i.d. complex Gaussian RVs, $\mathcal{C N}\left(0, N_{0}\right)$. Let $\lambda_{1}$ and $\lambda_{2}$ be the maximum eigenvalues of the complex central Wishart matrices $\mathbf{X}^{\dagger} \mathbf{X}$ and $\mathbf{Y}^{\dagger} \mathbf{Y}$, respectively, where $\mathbf{X}, \mathbf{Y}$ are $N_{2} \times N_{1}$ and $N_{3} \times N_{2}$ matrices with all standard complex Gaussian elements, $\mathcal{C N}(0,1)$. Then, the relationships between $\lambda_{\max _{1}}$ and $\lambda_{1}, \lambda_{\max _{2}}$ and $\lambda_{2}$ are given by $\lambda_{\max _{1}}=\Omega_{1}(1-$ $\left.\sigma_{e}^{2}\right) \lambda_{1}$ and $\lambda_{\text {max }_{2}}=\Omega_{2} \lambda_{2}$. For notational brevity, we utilize $\lambda_{3}$ to stand for $\left\|\mathbf{H}_{3}(t)\right\|_{F}^{2}$ and $\lambda_{4}$ to denote $\left\|\mathbf{H}_{4}(t)\right\|_{F}^{2}$. As a result, the expression for the end-to-end instantaneous SNR of the secondary network is obtained from (4), (5), (6) and (7), as

$$
\gamma_{D}=\frac{\lambda_{1} \lambda_{2}}{c \lambda_{2}+d \lambda_{2} \lambda_{3}+e \lambda_{1} \lambda_{4}}
$$

where $c=\frac{\left(2-\rho^{2}\right)}{\rho^{2}}, d=\frac{N_{0}}{Q \rho^{2} \Omega_{1}\left(1-\sigma^{2}\right)}$, and $e=\frac{N_{0}}{Q \Omega_{2}}$.

\section{End-To-End Performance AnALysis}

In this section, we analyze the outage probability (OP) and the symbol error rate (SER) of the considered system. To do so, we need to obtain the cumulative distribution function (CDF) of $\gamma_{D}$. However, deriving the exact expression of $F_{\gamma_{D}}(\gamma)$ from (8) is very challenging, so we use another approach. First, we propose a tightly bounded expression for the CDF of the instantaneous SNR, $\gamma_{D}$ in a similar manner as in [15, eq.(25)]. With this outcome, we will obtain tight expressions for the OP and SER of the considered system.

As mentioned in [14], the probability density function (PDF) of $\lambda_{1}$ is given by

$$
f_{\lambda_{1}}\left(\lambda_{1}\right)=K_{1} \sum_{k_{1}=1}^{P_{1}} \sum_{l_{1}=Q_{1}-P_{1}}^{\left(Q_{1}+P_{1}-2 k_{1}\right) k_{1}} d_{k_{1}, l_{1}} \lambda_{1}^{l_{1}} \exp \left(-k_{1} \lambda_{1}\right)
$$

where $P_{1}=\min \left(N_{1}, N_{2}\right), Q_{1}=\max \left(N_{1}, N_{2}\right)$, and $K_{1}^{-1}=$ $\prod_{i=1}^{P_{1}}\left(Q_{1}-1\right) !(i-1)$ !.
By integrating $f_{\lambda_{1}}(x)$ with respect to variable $x$ over the interval $\left(0, \lambda_{1}\right)$ and then applying [13, eq.(3.351.2)] to solve the integral, we obtain the CDF of $\lambda_{1}$ as

$$
\begin{aligned}
F_{\lambda_{1}}\left(\lambda_{1}\right) & =1-K_{1} \sum_{k_{1}=1}^{P_{1}} \sum_{l_{1}=Q_{1}-P_{1}}^{\left(Q_{1}+P_{1}-2 k_{1}\right) k_{1}} d_{k_{1}, l_{1}} l_{1} ! \sum_{m=0}^{l_{1}} \frac{k_{1}^{m}}{m !} \\
& \times \lambda_{1}^{m} \exp \left(-k_{1} \lambda_{1}\right)
\end{aligned}
$$

Similarly, the PDF and CDF of $\lambda_{2}$ are given by

$$
\begin{aligned}
f_{\lambda_{2}}\left(\lambda_{2}\right) & =K_{2} \sum_{k_{2}=1}^{P_{2}} \sum_{l_{2}=Q_{2}-P_{2}}^{\left(Q_{2}+P_{2}-2 k_{2}\right) k_{2}} d_{k_{2}, l_{2}} \lambda_{2}^{l_{2}} \exp \left(-k_{2} \lambda_{2}\right) \\
F_{\lambda_{2}}\left(\lambda_{2}\right) & =1-K_{2} \sum_{k_{2}=1}^{P_{2}} \sum_{l_{2}=Q_{2}-P_{2}}^{\left(Q_{2}+P_{2}-2 k_{2}\right) k_{2}} d_{k_{2}, l_{2}} l_{2} ! \sum_{n=0}^{l_{2}} \frac{k_{2}^{n}}{n !} \\
& \times \lambda_{2}^{n} \exp \left(-k_{2} \lambda_{2}\right)
\end{aligned}
$$

where $P_{2}=\min \left(N_{2}, N_{3}\right), Q_{2}=\max \left(N_{2}, N_{3}\right)$, and $K_{2}^{-1}=$ $\prod_{i=1}^{P_{2}}\left(Q_{2}-1\right) !(i-1) !$

Since $\lambda_{3}$ is the Frobenius norm of the channel coefficient matrix from $\mathrm{SU}_{\mathrm{TX}}$ to $\mathrm{SU}_{\mathrm{R}}$, it is a sum of the squares of $N_{4} \times N_{1}$ i.i.d. complex Gaussian RVs with zero mean and variance $\Omega_{3}$. Thus, $\lambda_{3}$ is a Gamma random variable with parameter set $\left(N_{1} N 4, \Omega_{3}\right)$ whose PDF and CDF are, respectively, written as

$$
\begin{aligned}
& f_{\lambda_{3}}\left(\lambda_{3}\right)=\frac{\lambda_{3}^{N_{1} N_{4}-1}}{\Omega_{3}^{N_{1} N_{4}} \Gamma\left(N_{1} N_{4}\right)} \exp \left(-\frac{\lambda_{3}}{\Omega_{3}}\right) \\
& F_{\lambda_{3}}\left(\lambda_{3}\right)=1-\exp \left(-\frac{\lambda_{3}}{\Omega_{3}}\right) \sum_{p=0}^{N_{1} N_{4}-1} \frac{\lambda_{3}^{p}}{\Omega_{3}^{p} p !}
\end{aligned}
$$

Similarly, $\lambda_{4}$ is a sum of the squares of $N_{4} \times N_{2}$ i.i.d. complex Gaussian RVs with zero mean and variance $\Omega_{4}$. Therefore, $\lambda_{4}$ has Gamma distribution with parameter set $\left(N_{2} N 4, \Omega_{4}\right)$ whose PDF, CDF are, respectively, given by

$$
\begin{aligned}
f_{\lambda_{4}}\left(\lambda_{4}\right) & =\frac{\lambda_{4}^{N_{2} N_{4}-1}}{\Omega_{4}^{N_{2} N_{4}} \Gamma\left(N_{2} N_{4}\right)} \exp \left(-\frac{\lambda_{4}}{\Omega_{4}}\right) \\
F_{\lambda_{4}}\left(\lambda_{4}\right) & =1-\exp \left(-\frac{\lambda_{4}}{\Omega_{4}}\right) \sum_{q=0}^{N_{2} N_{4}-1} \frac{\lambda_{4}^{q}}{\Omega_{4}^{q} q !}
\end{aligned}
$$

Now, we approximate $\gamma_{D}$ as in [15, eq.(25)], i.e, $\gamma_{D} \approx$ $\min \left(\gamma_{1}, \gamma_{2}\right)$ where $\gamma_{1}=\frac{\lambda_{1}}{c+d \lambda_{3}}$ and $\gamma_{2}=\frac{\lambda_{2}}{e \lambda_{4}}$. Because $\lambda_{1}, \lambda_{2}, \lambda_{3}, \lambda_{4}$ are independent, we can apply the order statistics theory to obtain the CDF of $\gamma_{D}$ as

$$
F_{\gamma_{D}}(\gamma)=1-\left[1-F_{\gamma_{1}}(\gamma)\right]\left[1-F_{\gamma_{2}}(\gamma)\right]
$$

where $F_{\gamma_{1}}(\gamma)$ and $F_{\gamma_{2}}(\gamma)$ are given by

$$
F_{\gamma_{1}}(\gamma)=\int_{0}^{\infty} F_{\lambda_{1}}\left(\gamma\left(c+d \lambda_{3}\right)\right) f_{\lambda_{3}}\left(\lambda_{3}\right) d \lambda_{3}
$$




$$
F_{\gamma_{2}}(\gamma)=\int_{0}^{\infty} F_{\lambda_{2}}\left(\gamma e \lambda_{4}\right) f_{\lambda_{4}}\left(\lambda_{4}\right) d \lambda_{4}
$$

Substituting (10), (13) into (18) and (12), (15) into (19), after some algebraic manipulations, we rewrite $F_{\gamma_{1}}(\gamma)$ and $F_{\gamma_{2}}(\gamma)$ as

$$
\begin{aligned}
& F_{\gamma_{1}}(\gamma)=1-K_{1} \sum_{k_{1}=1}^{P_{1}} \sum_{l_{1}=Q_{1}-P_{1}}^{\left(Q_{1}+P_{1}-2 k_{1}\right) k_{1}} \frac{d_{k_{1}, l_{1}} l_{1} !}{\Omega_{3}^{N_{1} N_{4}} \Gamma\left(N_{1} N_{4}\right)} \\
& \times \sum_{m=0}^{l_{1}} \frac{\gamma^{m}}{k_{1}^{l_{1}-m+1} m !} \sum_{u=0}^{m} C_{u}^{m} d^{u} c^{m-u} \exp \left(-k_{1} c \gamma\right) \\
& \times \int_{0}^{\infty} \lambda_{3}^{N_{1} N_{4}+u-1} \exp \left(-\frac{k_{1} \gamma d \Omega_{3}+1}{\Omega_{3}} \lambda_{3}\right) d \lambda_{3} \quad(20) \\
& F_{\gamma_{2}}(\gamma)= 1-K_{2} \sum_{k_{2}=1}^{P_{2}} \sum_{l_{2}=Q_{2}-P_{2}}^{\left(Q_{2}+P_{2}-2 k_{2}\right) k_{2}} \frac{d_{k_{2}, l_{2}} l_{2} !}{\Omega_{4}^{N_{2} N_{4}} \Gamma\left(N_{2} N_{4}\right)} \sum_{n=0}^{l_{2}} e^{n} \\
& \times \frac{\gamma^{n}}{k_{2}^{l_{2}-n+1} n !} \int_{0}^{\infty} \lambda_{4}^{N_{2} N_{4}+n-1} \exp \left(-\frac{k_{2} \gamma e \Omega_{4}+1}{\Omega_{4}} \lambda_{4}\right) d \lambda_{4}
\end{aligned}
$$

Utilizing [13, eq.(3.351.2)] to solve the integral of (20) and (21), the closed-form expressions for the CDF of $\gamma_{1}$ and $\gamma_{2}$ are acquired as

$$
\begin{aligned}
& F_{\gamma_{1}}(\gamma)=1-K_{1} \sum_{k_{1}=1}^{P_{1}} \sum_{l_{1}=Q_{1}-P_{1}}^{\left(Q_{1}+P_{1}-2 k_{1}\right) k_{1}} d_{k_{1}, l_{1}} l_{1} ! \sum_{m=0}^{l_{1}} \frac{1}{k_{1}^{l_{1}-m+1} m !} \\
& \times \sum_{u=0}^{m} C_{u}^{m} \frac{\Gamma\left(N_{1} N_{4}+u\right)}{\Gamma\left(N_{1} N_{4}\right)} \frac{d^{u} c^{m-u} \Omega_{3}^{u} \gamma^{m}}{\left(k_{1} \gamma d \Omega_{3}+1\right)^{N_{1} N_{4}+u}} \exp \left(-k_{1} c \gamma\right)
\end{aligned}
$$

$$
\begin{aligned}
F_{\gamma_{2}}(\gamma) & =1-K_{2} \sum_{k_{2}=1}^{P_{2}} \sum_{l_{2}=Q_{2}-P_{2}}^{\left(Q_{2}+P_{2}-2 k_{2}\right) k_{2}} d_{k_{2}, l_{2}} l_{2} ! \sum_{n=0}^{l_{2}} \frac{1}{k_{2}^{l_{2}-n+1} n !} \\
& \times \frac{\Gamma\left(N_{2} N_{4}+n\right)}{\Gamma\left(N_{2} N_{4}\right)} \frac{e^{n} \Omega_{4}^{n} \gamma^{n}}{\left(k_{2} \gamma e \Omega_{4}+1\right)^{N_{2} N_{4}+n}}
\end{aligned}
$$

By substituting (22) and (23) into (17), the CDF of the instantaneous end-to-end SNR $\gamma_{D}$ is finally obtained as

$$
\begin{aligned}
& F_{\gamma_{\mathrm{D}}}(\gamma)=1-K_{1} K_{2} \sum_{k_{1}=1}^{P_{1}} \sum_{l_{1}=Q_{1}-P_{1}}^{\left(Q_{1}+P_{1}-2 k_{1}\right) k_{1}} d_{k_{1}, l_{1}} l_{1} ! \sum_{m=0}^{l_{1}} \frac{1}{m !} \\
& \times \frac{1}{k_{1}^{l_{1}-m+1}} \sum_{u=0}^{m} C_{u}^{m} \sum_{k_{2}=1}^{P_{2}} \sum_{l_{2}=Q_{2}-P_{2}}^{\left(Q_{2}+P_{2}-2 k_{2}\right) k_{2}} d_{k_{2}, l_{2}} l_{2} ! \sum_{n=0}^{l_{2}} \frac{1}{n !} \\
& \times \frac{c^{m-u} d^{u} e^{n} \Omega_{3}^{u} \Omega_{4}^{n}}{k_{2}^{l_{2}-n+1}} \frac{\Gamma\left(N_{1} N_{4}+u\right)}{\Gamma\left(N_{1} N_{4}\right)} \frac{\Gamma\left(N_{2} N_{4}+n\right)}{\Gamma\left(N_{2} N_{4}\right)} \\
& \times \frac{\gamma^{m+n} \exp \left(-k_{1} c \gamma\right)}{\left(k_{2} \gamma e \Omega_{4}+1\right)^{N_{2} N_{4}+n}\left(k_{1} \gamma d \Omega_{3}+1\right)^{N_{1} N_{4}+u}}
\end{aligned}
$$

\section{A. Outage Probability}

Outage probability, the probability that the instantaneous SNR drops below a predefined threshold $\gamma_{t h}$, is easily obtained by using $\gamma_{t h}$ as argument of the CDF of the instantaneous SNR given in (24), $P_{\text {out }}=F_{\gamma_{D}}\left(\gamma_{\text {th }}\right)$.

\section{B. Symbol Error Rate}

As shown in [16], for several modulation schemes, the expression for SER can be derived directly from $F_{\gamma_{\mathrm{D}}}(\gamma)$ as

$$
P_{E}=\frac{a \sqrt{b}}{2 \sqrt{\pi}} \int_{0}^{\infty} F_{\gamma_{D}}(\gamma) \gamma^{-\frac{1}{2}} e^{-b \gamma} d \gamma
$$

where $a$ and $b$ are modulation parameters (see [16]), i.e., for $M$-PSK, $a=2, b=\sin ^{2}(\pi / M)$. By substituting (24) into (25), after some simplifications, the closed-form expression of a tight upper bound for the SEP is rewritten as

$$
\begin{aligned}
& P_{E}=\frac{a \sqrt{b}}{2 \sqrt{\pi}} \int_{0}^{\infty} \gamma^{-\frac{1}{2}} \exp (-b \gamma) d \gamma-\frac{a \sqrt{b} K_{1} K_{2}}{2 \sqrt{\pi}} \sum_{k_{1}=1}^{P_{1}} \\
& \times \sum_{l_{1}=Q_{1}-P_{1}}^{\left.Q_{1}+P_{1}-2 k_{1}\right) k_{1}} d_{k_{1}, l_{1}} l_{1} ! \sum_{m=0}^{l_{1}} \frac{1}{k_{1}^{l_{1}-m+1} m !} \sum_{u=0}^{m} C_{u}^{m} \\
& \times \sum_{k_{2}=1}^{P_{2}} \sum_{Q_{2}=Q_{2}-P_{2}}^{\left.l_{2}-2 k_{2}\right) k_{2}} d_{k_{2}, l_{2}} l_{2} ! \sum_{n=0}^{l_{2}} \frac{1}{k_{2}^{l_{2}-n+1} n !} d^{u} \\
& \times c^{m-u} e^{n} \Omega_{3}^{u} \Omega_{4}^{n} \frac{\Gamma\left(N_{1} N_{4}+u\right)}{\Gamma\left(N_{1} N_{4}\right)} \frac{\Gamma\left(N_{2} N_{4}+n\right)}{\Gamma\left(N_{2} N_{4}\right)} \\
& \times \int_{0}^{\infty} \frac{\gamma^{m+n-\frac{1}{2}} \exp \left(-\left(k_{1} c+b\right) \gamma\right)}{\left(k_{2} \gamma e \Omega_{4}+1\right)^{N_{2} N_{4}+n}\left(k_{1} \gamma d \Omega_{3}+1\right)^{N_{1} N_{4}+u}} d \gamma
\end{aligned}
$$

Utilizing [13, eq.(3.351.2)] to calculate the first integral of (26), and then applying the partial fraction in [13, eq.(3.326.2)] to transform the expression in the second integral of (26) into a tabulated form, we have

$$
\begin{aligned}
& P_{E}=\frac{a}{2}-\frac{a \sqrt{b} K_{1} K_{2}}{2 \sqrt{\pi}} \sum_{k_{1}=1}^{P_{1}} \sum_{l_{1}=Q_{1}-P_{1}}^{\left(Q_{1}+P_{1}-2 k_{1}\right) k_{1}} d_{k_{1}, l_{1}} l_{1} ! \\
& \times \sum_{m=0}^{l_{1}} \frac{1}{m !} \sum_{u=0}^{m} C_{u}^{m} \sum_{k_{2}=1}^{P_{2}} \sum_{l_{2}=Q_{2}-P_{2}}^{\left(Q_{2}+P_{2}-2 k_{2}\right) k_{2}} d_{k_{2}, l_{2}} l_{2} ! \sum_{n=0}^{l_{2}} \\
& \times \frac{1}{n !} \frac{1}{k_{1}^{N_{1} N_{4}+u+l_{1}-m+1} k_{2}^{N_{2} N_{4}+l_{2}+1}} \frac{c^{m-u}}{e^{N_{2} N_{4}} d^{N_{1} N_{4}}} \\
& \times \frac{1}{\Omega_{3}^{N_{1} N_{4}} \Omega_{4}^{N_{2} N_{4}} \frac{\Gamma\left(N_{1} N_{4}+u\right)}{\Gamma\left(N_{1} N_{4}\right)} \frac{\Gamma\left(N_{2} N_{4}+n\right)}{\Gamma\left(N_{2} N_{4}\right)}} \\
& \times\left[\sum_{i=1}^{N_{2} N_{4}+n} \kappa_{i} \int_{0}^{\infty} \frac{\gamma^{m+n-\frac{1}{2}} \exp \left(-\left(k_{1} c+b\right) \gamma\right)}{\left(\gamma+\frac{1}{k_{2} e \Omega_{4}}\right)^{i}} d \gamma\right. \\
& \left.+\sum_{j=1}^{N_{1} N_{4}+u} \theta_{j} \int_{0}^{\infty} \frac{\gamma^{m+n-\frac{1}{2}} \exp \left(-\left(k_{1} c+b\right) \gamma\right)}{\left(\gamma+\frac{1}{k_{1} d \Omega_{3}}\right)^{j}} d \gamma\right]
\end{aligned}
$$


where $\kappa_{i}$, and $\theta_{j}$ are defined as

$$
\begin{gathered}
\kappa_{i}=\left.\frac{d^{N_{2} N_{4}+n-i}\left[\left(\gamma+\frac{1}{k_{1} d \Omega_{3}}\right)^{-\left(N_{1} N_{4}+u\right)}\right]}{\left(N_{2} N_{4}+n-i\right) ! d \gamma^{N_{2} N_{4}+n-i}}\right|_{\gamma=-\frac{1}{k_{2} e \Omega_{4}}}(28) \\
\theta_{j}=\left.\frac{d^{N_{1} N_{4}+u-j}\left[\left(\gamma+\frac{1}{k_{2} e \Omega_{4}}\right)^{-\left(N_{2} N_{4}+n\right)}\right]}{\left(N_{1} N_{4}+u-j\right) ! d \gamma^{N_{1} N_{4}+u-j}}\right|_{\gamma=-\frac{1}{k_{1} d \Omega_{3}}}
\end{gathered}
$$

The integrals in (27) are solved by using [17, eq.(2.3 .6 .9)]. After some algebraic manipulations and re-arranging terms, we finally obtain an expression for the SER as follows:

$$
\begin{aligned}
& P_{E}=\frac{a}{2}-\frac{a \sqrt{b}}{2 \sqrt{\pi}} K_{1} K_{2} \sum_{k_{1}=1}^{P_{1}} \sum_{l_{1}=Q_{1}-P_{1}}^{\left(Q_{1}+P_{1}-2 k_{1}\right) k_{1}} d_{k_{1}, l_{1}} l_{1} ! \sum_{m=0}^{l_{1}} \frac{1}{m !} \\
& \times \sum_{u=0}^{m} C_{u}^{m} \sum_{k_{2}=1}^{P_{2}} \sum_{l_{2}=Q_{2}-P_{2}}^{\left(Q_{2}+P_{2}-2 k_{2}\right) k_{2}} d_{k_{2}, l_{2}} \sum_{n=0}^{l_{2}} \frac{l_{2} !}{n ! k_{1}^{N_{1} N_{4}+u+l_{1}-m+1}} \\
& \times \frac{c^{m-u} \Gamma\left(N_{1} N_{4}+u\right) \Gamma\left(N_{2} N_{4}+n\right) \Gamma\left(m+n+\frac{1}{2}\right)}{k_{2}^{N_{2} N_{4}+l_{2}+1} e^{N_{2} N_{4}} \Omega_{4}^{N_{2} N_{4}} d^{N_{1} N_{4}} \Omega_{3}^{N_{1} N_{4}} \Gamma\left(N_{1} N_{4}\right) \Gamma\left(N_{2} N_{4}\right)} \\
& \times\left[\sum_{i=1}^{N_{2} N_{4}+n} \kappa_{i} \frac{U\left(m+n+\frac{1}{2}, m+n+\frac{1}{2}+1-i, \frac{k_{1} c+b}{k_{2} e \Omega_{4}}\right)}{k_{2}^{m+n+\frac{1}{2}-i} e^{m+n+\frac{1}{2}-i} \Omega_{4}^{m+n+\frac{1}{2}-i}}\right. \\
& \left.+\sum_{j=1}^{N_{1} N_{4}+u} \theta_{j} \frac{U\left(m+n+\frac{1}{2}, m+n+\frac{1}{2}+1-j, \frac{k_{1} c+b}{k_{1} d \Omega_{3}}\right)}{k_{1}^{m+n+\frac{1}{2}-j} d^{m+n+\frac{1}{2}-j} \Omega_{3}^{m+n+\frac{1}{2}-j}}\right]
\end{aligned}
$$

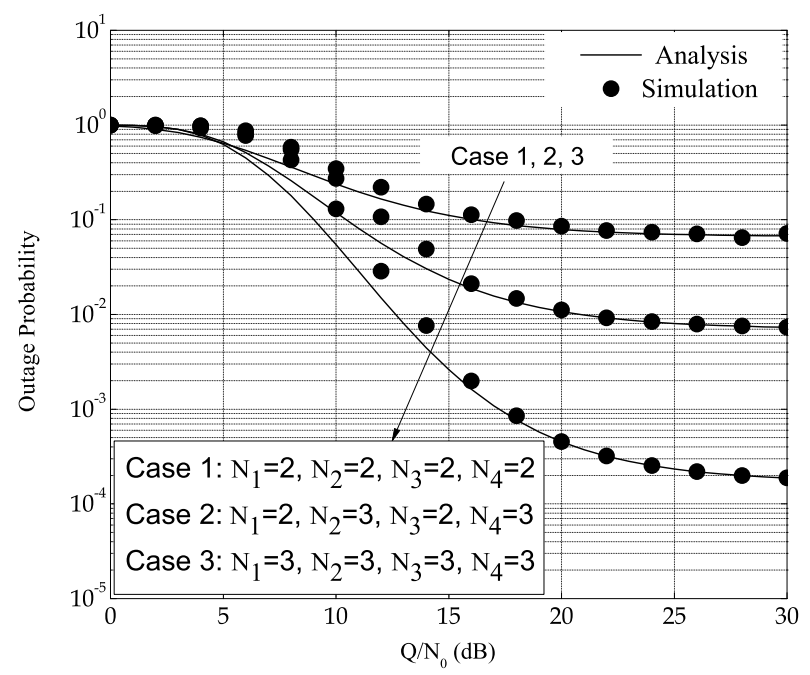

Fig. 2. Outage probability for cognitive AF relay systems with MRT for various transceiver antenna configurations.

\section{NUMERICAL RESULTS AND DISCUSSION}

In this section, numerical results are presented for the OP and SER of $Q$-PSK for the examined MRT scheme in a

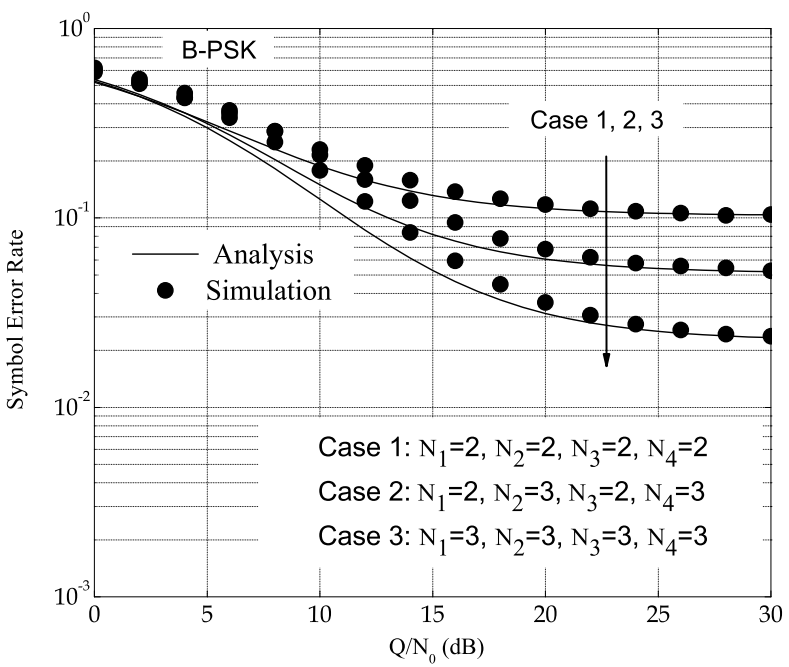

Fig. 3. SER of Q-PSK for cognitive AF relay systems with MRT for various transceiver antenna configurations.

cognitive AF relay network. In all scenarios, average channel power gains of Rayleigh fading channels are selected as $\Omega_{1}=0.5, \Omega_{2}=0.7, \Omega_{3}=0.8, \Omega_{4}=0.6$. First, we examine the effect of the number of transceiver antennas on the system by fixing the variance of CEE $\sigma^{2}=0.01$ and the normalized Doppler frequency $f_{d} \tau=0.03$ to scale the channel correlation coefficient $\rho=0.99$. Furthermore, to demonstrate the impact of CEE and FD on the considered system, we also illustrate the OP and SER when fixing the number of transceiver antennas while changing the level of channel estimation error and feedback delay.

Fig. 2 and Fig. 3 depict the OP and SER versus average SNR for various antenna configurations in three cases:

- Case 1: $\left(N_{1}, N_{2}, N_{3}, N_{4}=2,2,2,2\right)$

- Case 2: $\left(N_{1}, N_{2}, N_{3}, N_{4}=2,3,2,3\right)$

- Case 3: $\left(N_{1}, N_{2}, N_{3}, N_{4}=3,3,3,3\right)$

As can be seen from these figures, when the number of transceiver antennas increases, the OP and SER of the considered system is improved significantly; which illustrates the benefits of deploying MRT with multiple antennas in a cognitive AF relay system.

Fig. 4 and Fig. 5 plot the OP and SER versus average SNR for various levels of feedback delay and channel estimation error. We consider four examples where the system is equipped with $N_{1}=3$ transmit antennas at the $\mathrm{SU}_{\mathrm{TX}}, N_{2}=3$ receive antennas at the $\mathrm{SU}_{\mathrm{R}}, N_{3}=3$ receive antennas at the $\mathrm{SU}_{\mathrm{RX}}$, and $N_{4}=3$ receive antennas at the $\mathrm{PU}_{\mathrm{RX}}$. In Case 4 , the system has imperfect CSI in both channel estimation error $\sigma^{2}=0.01$ and feedback delay presented through the channel correlation coefficient $\rho=0.9$ (corresponding to $f_{d} \tau=0.1$ ). Case 5 has perfect channel estimation $\sigma^{2}=0$ but having feedback delay with $\rho=0.9$ or $f_{d} \tau=0.1$. Furthermore, in Case 6, we illustrate the system performance for channel estimation error $\sigma^{2}=0.5$ and no feedback delay $\rho=1.0$. 


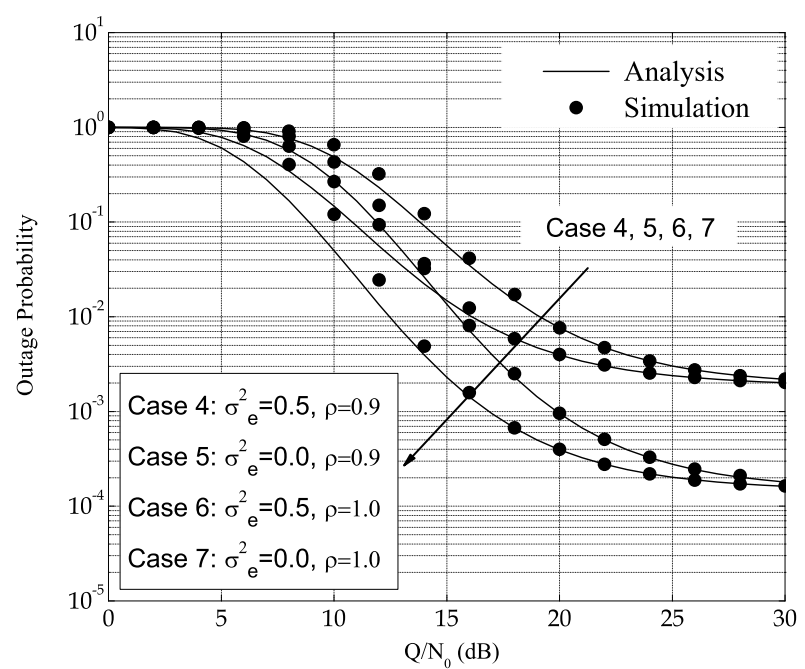

Fig. 4. Outage probability for cognitive AF relay systems with MRT for various levels of channel estimation error and feedback delay.

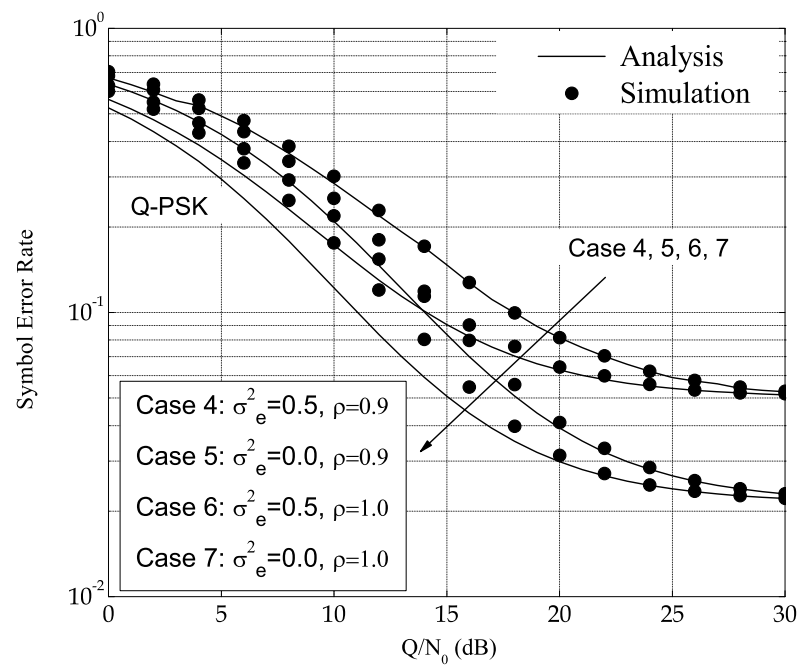

Fig. 5. SER of Q-PSK for cognitive AF relay systems with MRT for various levels of channel estimation error and feedback delay.

Finally, we present the OP and SER when $\mathrm{SU}_{\mathrm{R}}$ estimates the channel perfectly $\sigma^{2}=0$, and there is no feedback delay $\rho=0$ $\left(f_{d} \tau=0\right)$ in Case 7. As expected, the best performance can be achieved in Case 7 when having no channel estimation error and no feedback delay (perfect CSI). Conversely, the worst performance is obtained in Case 4 when having both channel estimation error and feedback delay. In the presence of feedback delay $\rho \neq 1$ as in Case 5 and channel estimation error $\sigma^{2} \neq 0$ as in Case 6, the system performance will be degraded, but not as serious as in Case 4. By comparing Case 4 with Case 5 and Case 6 with Case 7, the effect of CEE on the system will be observed. Further, we can notice that this influence is significant in low SNR and minor in the high SNR. Finally, by comparing Case 4 with Case 6 and Case 5 with Case 7, one can observe the impact of FD on the system performance being remarkable in all SNR range.

\section{CONCLUSION}

In this paper, we investigated the performance of an underlay cognitive AF relay system deploying MRT/MRC. More specifically, we have derived closed-form expressions in terms of tight upper bounds for OP and SER in i.i.d. Rayleigh fading channels. Our analysis takes into account the impact of both channel estimation error and feedback delay on the system. The numerical results illustrate the influence of the number of transceiver antennas and the effect of imperfect CSI including channel estimation error and feedback delay on the considered network.

\section{REFERENCES}

[1] Q. Zhang, J. Jia, and J. Zhang, "Cooperative relay to improve diversity in cognitive radio networks," IEEE Commun. Mag., vol. 47, no. 2, pp. 111-117, Feb. 2009.

[2] K. B. Letaief and W. Zhang, "Cooperative communications for cognitive radio networks," Proceedings of the IEEE, vol. 97, no. 5, pp. 878-893, May 2009.

[3] O. Simeone, J. Gambini, Y. Bar-Ness, and U. Spagnolini, "Cooperation and cognitive radio," in Proc. IEEE Int. Conf. Commun., Glasgow, Scotland, Jun. 2007, pp. 6511-6515.

[4] F. Negro, I. Ghauri, and D. T. M. Slock, "Beamforming for the underlay cognitive MISO interference channel via UL-DL duality," in Proc. of Crowncom, Cannes, Fance, Jun. 2010, pp. 1-5.

[5] V. A. Bohara, S. H. Ting, H. Yang, and A. Pandharipande, "Interferencefree overlay cognitive radio network based on cooperative space time coding," in Proc. of Crowncom, Cannes, France, Jun. 2010, pp. 1-5.

[6] R. C. Pereira, R. Souza, and M. Pellenz, "Overlay cognitive radio with multiple secondaries and its application to wireless mesh networks," in Proc. of IEEE Veh. Technol. Conf., Barcelona, Spain, Apr. 2009, pp. $1-5$.

[7] Y. Han, A. Pandharipande, and S. Ting, "Cooperative decode-andforward relaying for secondary spectrum access," IEEE Trans. Wireless Commun., vol. 8, no. 10, pp. 4945-4950, Oct. 2009.

[8] T. Duong, V. Bao, G. Alexandropoulos, and H.-J. Zepernick, "Cooperative spectrum sharing networks with AF relay and selection diversity," Electron. Lett., vol. 47, no. 20, pp. 1149-1151, Sep. 2011.

[9] M. S. Kaiser, "Power allocation for the network coded cognitive cooperative network," in Proc. of Wirelrss Commun. Conf., Wuhan, China, Sep. 2011, pp. 1-5.

[10] A. Louni and B. Khalaj, "Distributed beam-forming and power control in multi-relay underlay cognitive radio networks: a game-theoretical approach," in Proc. of Crowncom, Osaka, Japan, Jun. 2011, pp. 71-75.

[11] L. Li, X. Zhou, H. Xu, G. Y. Li, D. Wang, and A. Soong, "Simplified relay selection and power allocation in cooperative cognitive radio systems," IEEE Trans. Wireless Commun., vol. 10, no. 1, pp. 33-36, Jan. 2011.

[12] T. K. Y. Lo, "Maximum ratio transmission," IEEE Trans. Commun., vol. 47, no. 10, pp. 1458-1461, Oct. 1999.

[13] I. S. Gradshteyn and I. M. Ryzhik, Table of Integrals, Series, and Products, 7th ed. Academic Press, 2007.

[14] T. M. C. Chu, T. Q. Duong, and H.-J. Zepernick, "Outage probability and ergodic capacity for MIMO-MRT systems under co-channel interference and imperfect CSI," IEEE Swedish Commun. Techno. Workshop, Stockholm, Sweden, pp. 46-51, Sep. 2011.

[15] M. D. Renzo, F. Graziosi, and F. Santucci, "A comprehensive framework for performance analysis of dual-hop cooperative wireless systems with fixed-gain relays over generalized fading channels," IEEE Trans. Wireless Commun., vol. 8, no. 10, pp. 5060-5074, 2009.

[16] M. R. McKay, A. J. Grant, and I. B. Collings, "Performance analysis of MIMO-MRC in double-correlated Rayleigh environments," IEEE Trans. Commun., vol. 55, no. 3, pp. 497-507, Mar. 2007.

[17] A. P. Prudnikov, Y. A. Brychkov, and O. I. Marichev, Integrals and Series, vol. 1. New York: Gordon and Breach Science Publishers, 1986, vol. 1. 